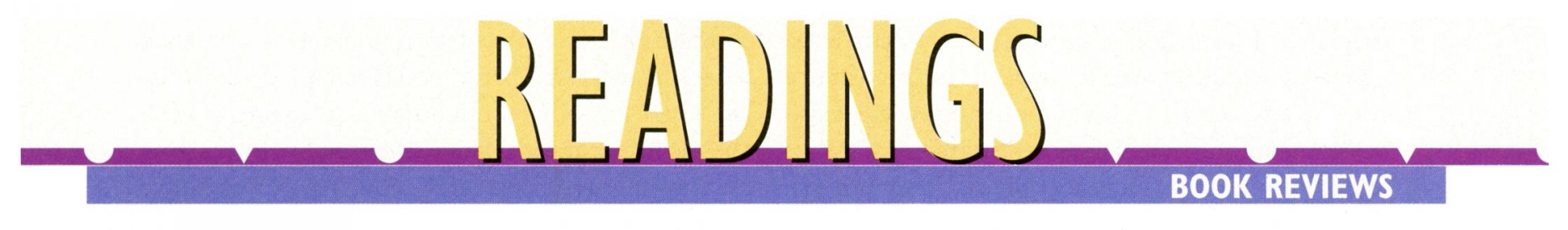

\title{
PREDICTABILITY OF WEATHER AND CLIMATE
}

Tim Palmer and Renate Hagedorn, Eds., 2006, 718 pp., \$170.00, hardbound, Cambridge University Press, ISBN 978-0-52I-84882-4

$\mathrm{t}$ was more than 40 years ago that Edward Lorenz published his paper on "Deterministic Nonperiodic Flow." This seminal paper spawned an impressive amount of research on the issue of predictability of the atmosphere-ocean system. Some developments that grew out of this research have subsequently been incorporated into operational weather predictionensemble forecasting being an important example. One of the mantras of the predict-

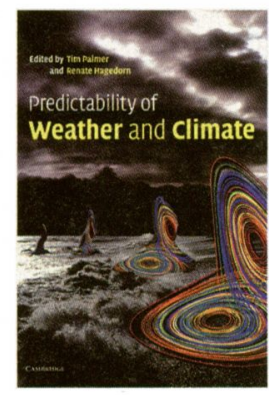
ability community is attributed to Tennekes and states that "no forecast is complete without a forecast of forecast skill."

Predictability of Weather and Climate provides a welcome review of the wealth and breadth of predictability research in recent decades. The book's 27 chapters-each written by leading researchers in the field-can be placed into three categories: theoretical, operational, and applied. The chapters are organized so as to provide an excellent primer in predictability and ensemble research, but each chapter is self-contained.

The first third of the book consists of chapters that discuss theoretical aspects of predictability and ensemble forecasting. These include a contribution from Edward Lorenz titled "Predictability-A Problem Partly Solved." This chapter was actually written in 1996, yet the title is still apt: while a significant amount of interesting work has been done in the field, there is still much to be done in developing a theory of predictability applicable to weather and climate systems. One of the most significant obstacles confronting researchers in the field is the "curse of dimensionality"-referring to the very high number of degrees of freedom of the systems under investigation. This typically prevents theoretical results from being directly applicable to operational systems. Low-dimensional systems-such as Lorenz's famous 1963 model-are useful to illustrate some of the issues, and several of the contributors use relatively low-dimensional systems in this way. Some of these examples would make interesting exercises for a graduate-level class in predictability, although the book is not intended to be a classroom text.

The theoretical chapters are a mix of mathematical discussions, such as the chapters on Liouville's equations and generalized stability theory, and also conceptual chapters on the origins of predictability from a dynamic meteorology perspective and on the philosophy of what predictability really means. The theory contributions do, however, remain well grounded in practicalities, and it is generally clear how the theory relates to operational issues of prediction.

The middle section of the book is more operationally oriented and includes descriptions of operational ensemble forecasting systems for short-, medium-, and longer-range forecasting. There is also discussion of ensemble prediction systems involving multiple models, as well as those in which initial conditions are perturbed. There is not, however, a comprehensive comparison of these two approaches, which is a shame, as their relative merits have been-and still are- a topic of discussion in the field. The book does contain a chapter that introduces the issue of model error in weather and climate models. This is one of the thorniest problems in the field as-unlike initial condition error and parametric error, which can be represented in a well-defined space given a model structure-there is no such space or metric for representing errors in the model structure itself.

Several of the chapters describing forecasting systems provide performance statistics. Unfortunately, little effort is made to even estimate sampling uncertainties in these statistics, let alone to subject hypotheses regarding prediction system performance to formal testing. It is possible that the apparent reluctance of meteorologists to use more rigorous statistical methods when evaluating forecast systems is a reflection of the deterministic 
philosophy that has dominated the field since the early part of the twentieth century. The omission of estimates of uncertainty on verification statistics is somewhat ironic for a field in which the importance of knowing uncertainty is so often emphasized; it also weakens some otherwise interesting and informative contributions about practical prediction methods. There is one chapter devoted to forecast evaluation, and the authors do mention the factors that create uncertainty in verification statistics. Hopefully, in the future, the importance of knowing the uncertainty in verification statistics will be as well appreciated as the importance of understanding the uncertainty in the forecasts themselves, and Tennekes's mantra will be joined by another: "No verification statistic is complete without an estimate of its uncertainty."

The final chapters of the book look at potential applications of ensemble prediction systems in decision support. Examples include the use of medium-range ensembles for predicting severe weather events, and the combined use of medium-range and seasonal forecasts for predicting monsoon rain. Several authors describe the "cost-loss" approach to making decisions with probabilistic forecasts, but unfortunately, specific examples involving real costs and real losses are absent. This is not a criticism of the book, but it is a symptom of a growing gap between what researchers know and what practitioners communicate. Although the limits of predictability have been appreciated since the 1960s, and ensemble forecasting has been used operationally for more than a decade, most weather forecasts remain deterministic. There are still few quantitative estimates of what the overall economic value of ensemble forecasting systems might be, although in fairness to ensemble forecasters, it should be pointed out that the same is true for traditional deterministic forecasting systems that have been around considerably longer.

Predictability of Weather and Climate should be read by any researcher new to the field, whether they are an established researcher in another area or a graduate student new to research. It is also valuable to those already actively involved in the field for the broad view it provides.

\section{-Mark Roulston}

Mark Roulston is a visiting research fellow in the Centre for the Analysis of Time Series at the London School of Economics.

\section{FOR FURTHER READING}

Lorenz, E. N., 1963: Deterministic nonperiodic flow. J. Atmos. Sci., 42, 433-471.

Tennekes, H., A. P. M. Baede, and J. D. Osteegh, 1987:

Forecasting forecast skill. Proc. ECMWF Workshop on Predictability. Reading, U.K., ECMWF, 277-302.

\section{NEW PUBLICATIONS}

\section{DARKENING PEAKS: GLACIER} RETREAT, SCIENCE, AND SOCIETY Ben Orlove, Ellen Wiegandt, and Brian H. Luckman, Eds., 2008, 282 pp., $\$ 45.00$, hardbound, University of California Press, ISBN 978-0-520-25305-6

On many mountains, bare, dark rock is now all that can be seen where snow and ice once stood-dramatic evidence of the accelerating pace of glacier retreat due to climate change. This work is the first to provide a multidisciplinary, global exploration of the scientific, social, and economic dimensions of this phenomenon. Bringing together contributors from five continents, it discusses the ways that scientists have observed and modeled glaciers, tells how climate change is altering their size and distribution, and looks closely at their effect on human life.
FLASH FLOODS IN TEXAS

Jonathan Burnett, 2008, 330 pp., $\$ 35.00$, hardbound, Texas A\&M University Press, ISBN 978-I-58544-590-5

After combing libraries and archives, interviewing county historians, visiting flood sites, and collecting scores of photographs, the author of this book presents 28 floods from around the state to create a narrative of a century of disastrous events. Beginning with the famous Austin dam break of 1900 and ending with the historic $\mathbf{2 0 0 2}$ flooding in the Hill Country, it chronicles the causes and courses of these catastrophic floods as well as their costs in material damage and human lives.
BREAKING OCEAN WAVES: GEOMETRY, STRUCTURE AND REMOTE SENSING

Eugene A. Sharkov, 2007, 278 pp., \$169.00, hardbound, Springer-Verlag, ISBN 978-3-540-29827-4

This book describes the physical findings of an investigation into the spatio-temporal characteristics of the gravity of breaking waves and the foam activity in open sea by methods and instruments of optical and microwave remote sensing. Emphasis is placed on the physical aspects of breaking processes necessary to measure the possibilities and limitations of remote sensing methods in specific observation cases of an oceanic surface. Numerous practical applications and illustrations are provided from airborne, shipborne, and laboratory experiments. 


\section{FUNDAMENTALS OF RADAR SIGNAL PROCESSING}

Mark A. Richards, 2005, 513 pp., $\$ 99.95$, hardbound, McGraw-Hill, ISBN 0-07-144474-2

S ince its development for observations of the weather, Doppler radar technology has proven to be one of the most important advancements in meteorological instrumentation for mesoscale and storm-scale studies. Over the coming years, the impact of radar will become even more significant through its use in data assimilation and numerical weather prediction efforts. The ability of electromagnetic waves to penetrate clouds and storms and to provide observations with high spatial and temporal resolution has transformed the field of weather radar into an important subdiscipline of meteorology. The interaction of electromagnetic waves with the atmosphere is the most fundamental aspect of radar meteorology, followed by radar design, signal processing, interpretation, and operational use. Fundamentals of

The book is extremely well organized beginning with chapter 1, where a concise review of radar history and the fundamental theory of radar systems are provided. As the title states, the book's emphasis is on signal processing and not electromagnetics, as is the case with many other treatments of radar. Nevertheless, a well-written overview of antenna theory (including phased arrays), receiver design, resolution considerations, and signal processing is provided in chapter 1 . In particular, I appreciated the connection made between

Fundamentals of Radar Signal Processing

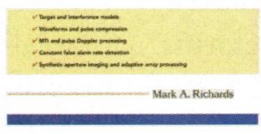
temporal and spatial sampling, which is important for an understanding of phased array antennas, for example.

Chapter 2 lays the groundwork for the rest of the book with definitions of important signal models, including amplitude models used to derive the radar range equation, frequency models for moving Radar Signal Processing, by Mark Richards, focuses on several important radar designs and their associated signal/array processing schemes. Although certainly targeted to an engineering audience, the book could serve as an in-depth treatment of the subject for meteorologists with the appropriate background in stochastic processes and signal theory. targets, and finally, both spatial and spectral models. Although briefly mentioned, one drawback of the book for the meteorological community is the lack of coverage of polarimetry radars, which will become only more important in the future with the planned polarimetric upgrade to the WSR-88D network.
FORESTRY AND CLIMATE CHANGE

P. H. Freer-Smith, M. S. J. Broadmeadow, and J. M. Lynch, 2007, 260 pp., \$140.00, hardbound, Oxford University Press, ISBN 978-I-84593-294-7

Emerging as a component of the international dialogue on the environment and climate, the role of forests in influencing Earth systems will need to be assessed. Drawing together perspectives from researchers and policy makers, this book explores how forests will interact with the physical and natural world, and with human society, as the climate changes. Also considered is how the world's forests can be managed to contribute to the mitigation of climate change and to maximize the full range of economic and nonmarket benefits.
CLIMATE EXTREMES AND SOCIETY Henry F. Diaz and Richard J. Murnane, Eds., 2008, 356 pp., $\$ 140.00$, hardbound, Cambridge University Press, ISBN 978-0-521-87028-3

Ordinary citizens, the insurance industry, and governments are concerned about the apparent increase in the frequency of weather and climate events causing extreme-and in some instances, catastrophic-impacts. This title focuses on the recent and potential future consequences of weather and climate extremes for different socioeconomic sectors. It also examines actions that may enable society to better respond to climate variability, and it provides examples of the impact of climate and weather extremes on society.
ATMOSPHERIC ACOUSTIC REMOTE SENSING

Stuart Bradley, 2007, 296 pp., \$119.95, hardbound, CRC Press, ISBN 978-0-8493-3588-4

Sonic Detection and Ranging (SODAR) systems and Radio Acoustic Sounding Systems (RASS) use sound waves to determine wind speed, wind direction, and turbulent character of the atmosphere. This book explains the underlying science, principles, and operational aspects of acoustic radars. Guidelines and tools are provided for handling calibration, error, equipment, hardware, sampling, and data analysis. The final chapter explores applications in environmental research, boundary layer research, wind power and loading, complex terrain, and sound speed profiles. 
Chapter 3 provides an introduction to the concept of the data cube, which generalizes the sampling of the radar signal in fast time (range time), slow time (sample time), and in space, or phase center. Sampling is often misunderstood but is a reality of any pulsed Doppler weather radar. Richards's presentation is well thought out and unifies these disparate sampling regimes. Quantization is also covered in this chapter and completes the background needed to begin a study of the important and timely topic of digital receiver technology.

Most current Doppler weather radars use a highpowered transmitter modulated by a simple pulse waveform. However, new designs are emerging that exploit pulse compression techniques in order to reduce peak-power requirements. Two of the more important candidate techniques (linear frequency modulation and binary pulse coding) are presented in chapter 4 under the general topic of radar waveforms. Limitations and design criteria are examined in the context of signal-to-noise ratio (SNR), resolution
(Doppler and range), and ambiguities. The matched filter is discussed as a means of maximizing SNR for different waveforms.

With the advent of more powerful processors on weather radars, spectral analysis is now a real possibility for the development of advanced signal processing techniques. For example, ground clutter filters have recently been developed that exploit the distinct advantages of examining the signal in the frequency domain using the Doppler spectrum. The book provides an excellent introduction to Doppler processing in chapter 5 beginning with the Moving Target Indication (MTI) method used in tracking radar. Although not used in the weather radar community, many of the concepts are similar to conventional ground clutter filters, and it was interesting to see the connection. In this chapter, the pulse pair processor is introduced, highlighting the relevance of the text to weather radars. Also provided in this chapter is a brief discussion of ground clutter filtering techniques for moving (airborne) radar platforms.

Chapters 6 and 7 provide a succinct coverage of detection theory, which is fundamentally important for aircraft surveillance/tracking radar, with its roots in communication theory. Although not directly used in current weather radar applications (with the possible exception of detection of point target contamination), the topic could become more important to the weather radar community if multimission capabilities become a reality.

A breakthrough in spatial resolution of airborne and spaceborne radar was achieved with the advent of synthetic aperture radar (SAR), which is covered in chapter 8 . By exploiting a moving platform and a stationary target, the effective aperture of the radar system can be increased, providing improved angular resolution. The book provides an introductory but excellent overview of the fundamental theory and techniques behind SAR. More applicable to observations of terrain and oceans, SAR has not seen much usage in 
meteorological studies. Nevertheless, this important and innovative method is a precursor, and provides a logical introduction to the topics in the next chapter on phased array radar.

The book ends with concise but comprehensive coverage of the fundamental theory of both conventional and adaptive beamforming algorithms used with phased array radars, exploiting the spatial dimension of the data cube. Certainly, phased array radar is an advanced topic for the meteorological radar community. However, these radars may become more relevant over the coming years with the design of the next-generation national weather radar network presently being contemplated. Significant advantages exist for weather observations using phased arrays, including improved temporal resolution, adaptive sensing and clutter rejection, crossbeam wind estimation, elimination of beam smearing, and possible multimission applications. However, challenges are also present that must be addressed before such advanced radar systems will become practical for operational weather radar networks.

In summary, I found the book by Richards extremely well organized, helping to unify a range of topics under one mathematical framework. Although numerous other texts exist on the subject of radar (e.g., Skolnik's Introduction to Radar Systems) - and to a lesser extent weather radar-this indepth treatment of signal processing fills an important niche. One drawback of the book, from the perspective of a professor, is the lack of any theoretical or computational problem sets. Nevertheless, the book represents a significant contribution to the radar community and will serve as an indispensable reference for years.

-Robert D. PAlMer

Robert Palmer is a professor and holds the Tommy C. Craighead Chair in the School of Meteorology at the University of Oklahoma $(\mathrm{OU})$. He directs the interdisciplinary Atmospheric Radar Research Center (ARRC) at OU and is deeply interested in all aspects of radar for studies of the atmosphere.

\section{FOR FURTHER READING}

Bringi, V. N., and V. Chandrasekhar, 2001: Polarimetric Doppler Weather Radar: Principles and Applications. Cambridge University Press, $636 \mathrm{pp}$.

Doviak, R. J., and D. S. Zrnic, 2006: Doppler Radar and Weather Observations. 2d ed. Dover, 562 pp.

Rinehart, R. E., 2004: Radar for Meteorologists. Rinehart Publishing, $482 \mathrm{pp}$.

Skolnik, M. I., 2001: Introduction to Radar Systems. McGraw-Hill, $772 \mathrm{pp}$.

Stoica, P., and R. Moses, 2005: Spectral Analysis of Signals. Prentice Hall, 452 pp.

Weber, M. E., J. Y. N. Cho, J. S. Herd, J. M. Flavin, W. E. Benner, and G. S. Torok, 2007: The next-generation multimission U.S. surveillance radar network. Bull. Amer. Meteor. Soc., 88, 1739-1751.

\section{REANALYSIS}

Looking back at "News and Notes" in the Bulletin of August 1980:

\section{NASA Tests Solar-Powered Engine/Generator for SMaller Users}

A joint government/industry team will design, fabricate, and test a solarpowered, $20 \mathrm{~kW}$ engine/generator designed for use by small communities, industries, farms, military bases, and similar users. The project, sponsored by the U.S. Department of Energy, is designed to foster effective and inexpensive methods of using solar energy to produce electric power and industrial heat. ...

Solar energy to power the engine/generator would be focused by a dishshaped parabolic mirror into a receiver where the heat is absorbed to run the engine. The engine/generator unit includes a turbine, compressor, alternating current generator, and gearbox selected from commercially available components. To increase energy efficiently, the engine will include a recuperator through which heat in the turbine exhaust is returned to the engine cycle. The engine/generator also would be equipped with a combustor so that power can continue to be produced at night and when skies are overcast.

The operation of this solar Brayton engine starts with the compressor, which takes in surrounding air and delivers it to a recuperator at about three times atmospheric pressure. In the recuperator the high-pressure air absorbs waste heat from the turbine exhaust and goes on to the solar receiver where it is further heated to $\sim 800^{\circ} \mathrm{C}$. The high-pressure, high-temperature air is used to drive the turbine, which produces sufficient power to generate electricity and drive a compressor. When the Sun's intensity drops, the cooler air leaving the receiver is heated to $\sim 800^{\circ} \mathrm{C}$ in a combustor placed in the ducting to the turbine.

The solar thermal generator could perform as the user's primary source of electric power or could be connected to an existing utility grid....

-Bull. Amer. Meteor. Soc., 61, 917 


\section{GLOSSARYOF}

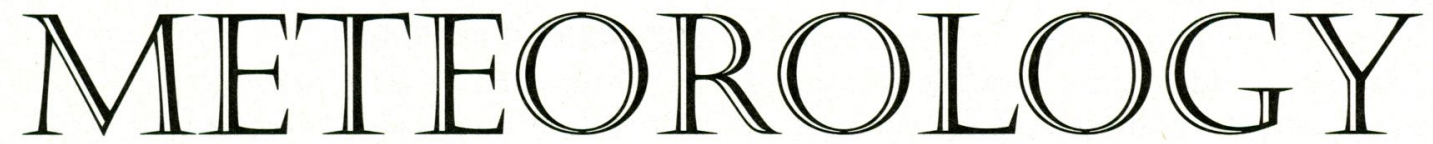

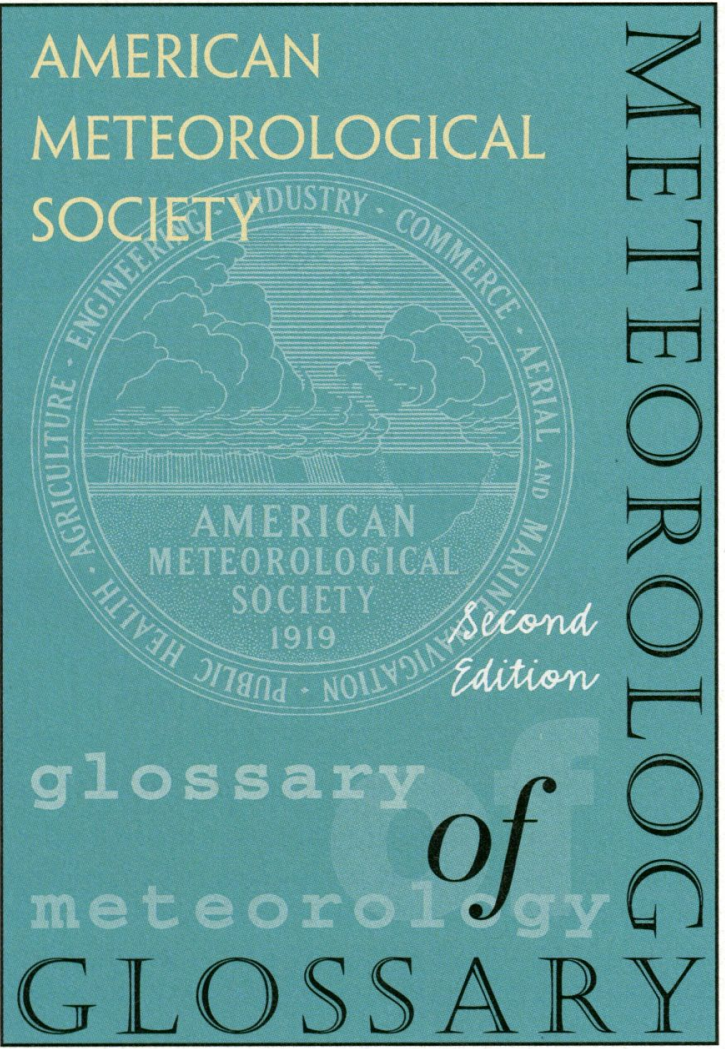

\section{Second Edition}

In 1959, the AMS published the Glossary of Meteorology. Containing 7900 terms, more than 10,000 copies have been sold over four decades through five printings. It is a tribute to the editors of the first edition that it has withstood the test of time and continued to be among the leading reference sources in meteorology and related sciences.

Now, over five years in the making, the second edition is available. The volume contains over 12,000 terms, including those from "new" disciplines, such as satellite meteorology and numerical weather prediction. In addition, related oceanographic and hydrologic terms are defined.

The Glossary of Meteorology, Second Edition, was produced by an editorial board comprised of 41 distinguished scientists and the participation of over 300 contributors. The CD-ROM version is compatible with Windows, Macintosh, and most UNIX platforms, and features hyperlinked cross-references.
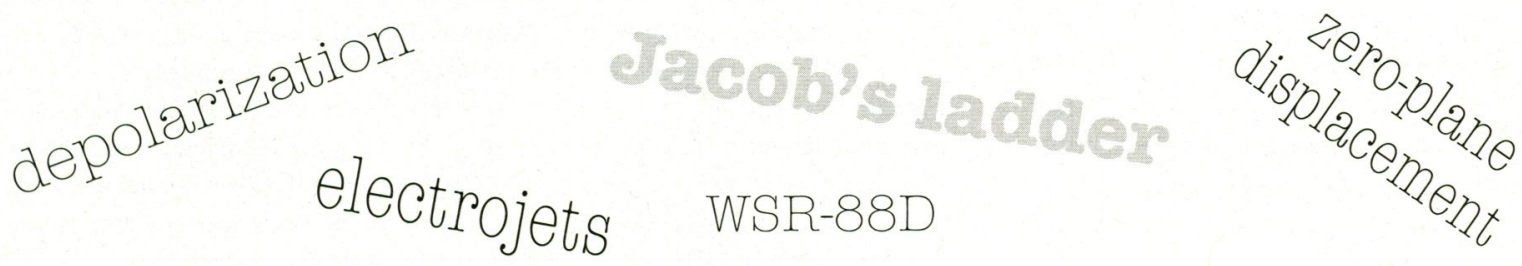

ISBN 1-878220-34-9, approx. 850 pp., hardbound. To place an order, refer to the pricing chart below and submit your prepaid orders to: Order Department, AMS, 45 Beacon Street, Boston, MA 02108-3693; call 617-227-2425 to order by phone using Visa, Mastercard, or American Express; or send e-mail to amsorder@ametsoc.org. Please make checks payable to the American Meteorological Society.

HARDBOUND BOOK

$\$ 85$ List

$\$ 60$ AMS Members

\$35 AMS Student Members
CD-ROM

$\$ 95$ List

\$65 AMS Members

\$35 AMS Student Members

\section{heiligenschein}

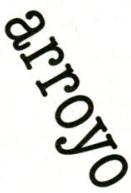

